Western University

Scholarship@Western

Robarts Imaging Publications

Robarts Research Institute

2015

\title{
Magnetic resonance imaging and histology correlation in the neocortex in temporal lobe epilepsy.
}

Maged Goubran

Robert RHammond

Sandrine de Ribaupierre

Jorge G Burneo

Seyed Mirsattari

See next page for additional authors

Follow this and additional works at: https://ir.lib.uwo.ca/robartspub

Part of the Bioimaging and Biomedical Optics Commons

Citation of this paper:

Goubran, Maged; Hammond, Robert R; de Ribaupierre, Sandrine; Burneo, Jorge G; Mirsattari, Seyed; Steven, David A; Parrent, Andrew G; Peters, Terry M; and Khan, Ali R, "Magnetic resonance imaging and histology correlation in the neocortex in temporal lobe epilepsy." (2015). Robarts Imaging Publications. 20.

https://ir.lib.uwo.ca/robartspub/20 


\section{Authors}

Maged Goubran, Robert R Hammond, Sandrine de Ribaupierre, Jorge G Burneo, Seyed Mirsattari, David A Steven, Andrew G Parrent, Terry M Peters, and Ali R Khan 


\section{MRI and histology correlation in the neocortex of temporal lobe epilepsy}

Maged Goubran 1,2,*, Robert R. Hammond ${ }^{4}$, Sandrine de Ribaupierre ${ }^{3}$, Jorge G. Burneo ${ }^{3}$, Seyed Mirsattari $^{3}$, David Steven ${ }^{3}$, Andrew G. Parrent ${ }^{3}$, Terry M. Peters ${ }^{1,2,5}$, Ali R. Khan ${ }^{1,5}$

${ }^{1}$ Imaging Research Laboratories, Robarts Research Institute,

${ }^{2}$ Biomedical Engineering Graduate Program,

${ }^{3}$ Epilepsy Program, Department of Clinical Neurological Sciences,

${ }^{4}$ Department of Pathology, Division of Neuropathology,

${ }^{5}$ Department of Medical Biophysics,

Western University, London, Ontario, Canada

\section{* Corresponding author}

Maged Goubran

mgoubran@robarts.ca

(519) 719-6705

P.O Box 50151151 Richmond St. North, London, Ontario, Canada, N6A 5B7

\section{Running Title}

MRI-histology correlation in focal epilepsy

Number of characters in title: 72

Number of characters in running title: 44

Number of Figures: 7

Number of colour figures: 5

Word count (abstract): X

Word count (manuscript): $\mathrm{X}$

Number of tables: 3 


\section{$\underline{\text { Abstract }}$}

\section{Objective}

To investigate the histopathological correlates of quantitative relaxometry and DTI and determine their efficacy in epileptogenic lesion detection for pre-operative evaluation of focal epilepsy.

\section{Methods}

We correlated quantitative relaxometry and DTI with histological features of neuronal density and morphology in 55 regions of the temporal lobe neocortex, selected from 13 patients who underwent epilepsy surgery. We made use of a validated non-rigid image registration protocol to obtain accurate correspondences between in-vivo MRI and histology images.

\section{Results}

We found T1 to be a predictor of neuronal density in the neocortical GM using linear mixed effects models with random effects for subjects. FA was a predictor of neuronal density of largecaliber neurons only (pyramidal cells, layers 3/5). Comparing multivariate to univariate mixed effects models with nested univariate demonstrated that employing T1 and FA together provided a significantly better fit than $\mathrm{T} 1$ or FA alone in predicting density of large-caliber neurons. Correlations with clinical variables revealed significant positive correlations between neuronal density with age $\left(\mathrm{r}_{\mathrm{s}}=0.726, \mathrm{p}_{\mathrm{fwe}}=0.021\right)$. This study is the first to relate in-vivo T1 and FA values to the proportion of neurons in GM.

\section{Interpretation}

Our results suggest that quantitative T1 mapping and DTI may have a role in pre-operative evaluation of focal epilepsy and can be extended to identify gray matter pathology in a variety of neurological disorders.

\section{Key words}

Temporal lobe epilepsy, MRI, histology, correlation, relaxometry, neuronal density 


\section{Introduction}

2 Approximately $30 \%$ of epileptic patients do not achieve remission with drugs ${ }^{1}$. Temporal lobe epilepsy (TLE) is the most common form of intractable focal epilepsy 2 and for many of these

4 patients the standard of care is surgical treatment. A randomized controlled trial has shown this

5 to be an effective treatment 3. However, seizure outcomes following surgical resection remain

6 suboptimal, with a recent long-term study demonstrating that only half of such patients are

7 seizure-free after 10 years 4 . It is believed that early seizure recurrence is due to inadequate identification or removal of the epileptic lesion(s) or network 5, which may suggest the presence

9 of dual pathology (histological abnormalities in the neocortex of patients with hippocampal sclerosis) or error in localising subtle neocortical lesions. However, whether these residual

11 abnormalities are epileptogenic, or instead are the result of recurrent seizures, is still unclear.

In addition to electroencephalography, MRI can identify lesions related to seizure onset, and surgical outcomes are more favorable if an underlying lesion can be detected ${ }^{6,7}$. However, clinical protocols for pre-operative assessment of focal epilepsy lack sensitivity, with more than $30 \%$ of patients diagnosed as MRI negative ${ }^{8,9}$, and the histological evaluation often reveals

17 reactive changes or malformations of cortical development (MCD) ${ }^{10,11}$. Quantitative MRI sequences and image processing techniques such as T2 relaxometry mapping, diffusion tensor 19 imaging (DTI), voxel-based morphometry and cortical thickness can reveal subtle pathologies 20 undetected on routine MRI ${ }^{12,13,14}$.

21 Imaging-histopathological correlations studies from neocortical specimens in TLE have

23 been used to better understand the relationships between the two. Garbelli et al. (2012) 15 
24 demonstrated that blurred cortical boundaries in the temporal pole is correlated to degeneration 25 of fibre bundles. With visually-matched ROIs, Eriksson et al. (2007) ${ }^{16}$ found a negative 26 correlation between GM fast FLAIR T2 (FFT2) and neuronal nuclear antigen (NeuN). A follow-

27 up study ${ }^{17}$ investigating GM probability maps with NeuN and glial fibrillary acidic protein 28 (GFAP) did not find any correlations. Similarly, another study ${ }^{18}$ also incorporating FLAIR and 29 DTI still failed to find any correlations. Such data suggest that the pathological basis of abnormal MRI signals is poorly understood in focal epilepsy. The study and identification of quantitative

31 imaging correlates relating to neocortical abnormalities can potentially reveal the association

32 between these specific MRI parameters and seizure outcomes in MRI-negative patients. It would 33 allow, as well the investigation of their effects on long-term surgical outcomes of patients with 34 hippocampal sclerosis.

37 quantitative relaxometry and DTI from neocortical specimens of intractable TLE patients. We 38 make use of a validated non-rigid image registration protocol to obtain accurate correspondences 39 between quantitative in-vivo MRI and histology images. We first sample quantitative histology 40 parameters from the gray and white matter in each $\mathrm{NeuN}$ (representing neuron integrity) and 41 GFAP (representing gliosis) slide, and then use image registration to obtain the corresponding 42 MRI parameters from high-resolution quantitative T1 and T2 maps along with DTI.

$44 \quad$ Materials and methods

$45 \quad$ Patients and Samples 
Our study cohort included 13 TLE (5 males, 8 females, age: $34 \pm 15$ (range: 18-56)) who

47 underwent anterior temporal lobectomy (ATL) surgery. This project, part of an ongoing research

67

study at the Robarts Research Institute, was approved by the office of research and ethics of Western University, and informed consent was obtained from all patients prior to their recruitment in the study. Patients had preoperative investigations including neuropsychological testing and 1.5T clinical MRI scans, which included T1-weighted, T2-weighted, FLAIR, and diffusion-weighted sequences. Patients were monitored with video-scalp EEG telemetry for seizure characterization, with three patients requiring subdural electrodes placement. In addition to the $1.5 \mathrm{~T}$ clinical MRI scans performed as part of their clinical diagnosis, patients underwent a series of scans on a 3T MRI research scanner as described in the in-vivo MRI imaging subsection. Table 1 summarizes the age at the time of the last consultation prior to surgery, gender, age at seizure onset, electrographic seizure origin as well as clinical MRI and pathology findings for our patient cohort.

\section{In-vivo Magnetic Resonance Imaging}

All patients underwent pre-operative imaging, comprising relaxation mapping and DTI, on a 3 Tesla Discovery MR750 scanner (General Electric, Milwaukee, WI, U.S.A.) with a 32 channel head coil. For T1 mapping we employed the DESPOT1-HIFI approach ${ }^{19}$ which involves the acquisition of two 3D SPGR sagittal T1-weighted image volumes $(\mathrm{TR}=8.36 \mathrm{~ms}, \mathrm{TE}=3.71 \mathrm{~ms}$, flip angles $=4^{\circ} \& 18^{\circ}$, matrix $=220 \times 220$, slice thickness $=1 \mathrm{~mm}, \mathrm{FOV}=220 \mathrm{~mm}$ ), as well as an additional inversion-prepared SPGR for B1 mapping $\left(\mathrm{TR}=6.4 \mathrm{~ms}, \mathrm{TE}=3.1 \mathrm{~ms}\right.$, flip angle $=5^{\circ}$, matrix $=220 \times 128$, slice thickness $=1 \mathrm{~mm}, \mathrm{FOV}=220 \mathrm{~mm}$ ). For $\mathrm{T} 2$ mapping the DESPOT2-FM approach ${ }^{20}$ was used, whereby five balanced steady-state free precession (bSSFP) images were 
acquired with flip angles $5^{\circ}, 35^{\circ}$ and $68^{\circ}$ with phase cycling patterns $\theta_{\mathrm{RF}}=0^{\circ}$ and $180^{\circ}$ $(\mathrm{TR}=4.6 \mathrm{~ms}, \mathrm{TE}=2.3 \mathrm{~ms}$, matrix $=220 \times 220$, slice thickness $=1, \mathrm{FOV}=220 \mathrm{~mm}) . \mathrm{DTI}$ was performed using an axial spin-echo echo-planar imaging (EPI) sequence with 41 diffusion directions and a b-value of $1000\left(\mathrm{TR}=1100 \mathrm{~ms}, \mathrm{TE}=63.2 \mathrm{~ms}\right.$, flip angle $=90^{\circ}$, matrix $=96 \times 96$, slice thickness $=2.5$, $\mathrm{FOV}=240 \mathrm{~mm})$. To compute $\mathrm{T} 1$ and $\mathrm{T} 2$ quantitative maps, all the weighted images were registered to the first scan of the session using the FLIRT tool of the FSL image analysis suite (FSL, http://fsl.fmrib.ox.ac.uk) with an affine transformation to correct for motion between scans. T1 and T2 quantitative maps were subsequently reconstructed from their respective weighted images using their signal equations as described in (Deoni et al., 2007, 2009) ${ }^{19,}{ }^{.}$ Eddy-current correction and diffusion tensor estimation were performed using FMRIB's Diffusion Toolbox (FDT) and maps of fractional anisotropy (FA), mean diffusivity (MD), radial diffusivity (RD) and axial diffusivity (AD) were transformed and resampled to the coordinate system defined by the $1 \mathrm{~mm}$ isotropic T1 map.

\section{Histological processing and quantitative histology}

The specimens underwent accessioning and gross examination at the Department of Pathology at the University Hospital of London Health Sciences Centre, and were then bissected in the coronal plane. Each half of the specimen was embedded in agar for support and stabilization during slicing. The half-specimens were then sectioned, parallel to the initial cut, into $4.4 \mathrm{~mm}$ thick coronal slices using a commercial deli slicer. Each block was embedded in paraffin and sectioned at a thickness of $8 \mu \mathrm{m}$. Slides from each block were stained with hematoxylin and eosin (H\&E) and processed for immunohistochemistry (IHC) to examine for NeuN (monoclonal antibody) and GFAP (polyclonal antibody) expression. Batch IHC processing was performed on 
92 a Dako Autostainer Link 48 (Dako Corporation, Glostrup, Denmark) to minimize variability

93 between slides. The resulting slides were digitized on a ScanScope GL (Aperio Technologies,

94 Vista, CA, USA) bright field slide scanning system at a maximum of 20x optical zoom, and

95 stitched to form full-frame multi-resolution images stored in BigTIFF file format (maximum

96 pixel resolution $0.5 \mu \mathrm{m})$.

97

Field fraction estimates (proportion of all pixels in the field that were positively-stained)

99

100

101

102

103

104

105

106

107

108

109

110

111

112

113

114

were used to quantify the NeuN and GFAP IHC. These estimates have been used in previous studies to represent neuronal integrity and gliosis ${ }^{16,17,18}$ and are sensitive to the packing density and cell-size of neuronal cell bodies and processes (NeuN) or astrocytes (GFAP). The positive pixel count algorithm (Aperio Technologies, Vista, CA, USA) was employed for this purpose and employs color-based thresholding for hue, saturation, and intensity to determine whether or not a pixel is immuno-positive. Slides were batch processed using scripts written in MATLAB (The MathWorks Inc., Natick, MA, USA), processing the full resolution images in blocks of 100um x 100um. Hue and saturation thresholds were fixed (Hue value $=0.1$, Hue width $=0.2$ and saturation $=4 \times 10^{-2}$ ) and the intensity threshold was chosen for each case to visualize the immuno-positive pixels and account for staining variability between slides.

Field fraction measurements involving dysplastic cortex could be less sensitive when reductions in packing density are accompanied by cyto-morphological size changes, since each would affect the field fraction in opposing directions. To better decouple these factors, we developed a method for segmenting the neuronal cell bodies to provide local estimates of neuron density and size. This procedure first employs colour deconvolution ${ }^{21}$ to extract the colour 
115 component related to immuno-positive staining, then performs a watershed-based segmentation

116 procedure $^{22}$ for splitting joined or connected neurons, removes objects smaller than a predefined

117 area defined as noise (less than $14 \mu^{2}$ ). This provides a segmentation of each individual neuron

118 cell body that can be used to determine the neuron density in this field (\# of neurons/field) and

119 the mean size of neuron cell bodies in the field. To further discriminate between neurons, we also

120 categorized them as either small-calibre (granular cells) or large-calibre (pyramidal cells) using

121 an area threshold of $125 \mu^{2}$ and reported the density of each of these in the field. This

122 procedure inherently allows analysis for laminar specificity since larger neurons are typically

123 found in layers 3 and 5, and smaller neurons in layer 2 and 4. NeuN slides were batch-processed

124 with scripts written in MATLAB, to extract the neuron-specific quantitative features in each

$125100 \mu \mathrm{m} \times 100 \mu \mathrm{m}$ field. Figure 1 illustrates this procedure and demonstrates the six different

126 quantitative histological features: NeuN field fraction, neuron density, mean neuron size, small

127 neuron density, large neuron density, and GFAP field fraction.

128

129 ROI Placement and Image Registration

130 To quantitatively correlate in-vivo MRI parameters and corresponding histological features, we

131 relied on region of interest (ROI) analysis as a means of extracting the desired parameters and

132 features from homologous regions. Histology ROIs were delineated on $100 \mu \mathrm{m}$ downsampled

133 H\&E histology slices using ITKsnap ${ }^{23}$. Since the middle temporal gyrus was present in all

134 available resections, ROIs were defined on the histology slides at the crown of the gyrus

135 comprising gray matter (GM) and white matter (WM) sub-regions (Figure 2), as was also done

136 by Eriksson et al. (2007) ${ }^{16}$. The edges of the WM ROIs were constrained to be $2 \mathrm{~mm}$ from the

137 gray/white boundary and were not delineated inside the high curvature regions of the gyrus. The 
138 boundaries of the GM ROI were limited to a distance of $1 \mathrm{~mm}$ from the pia to avoid partial

139 volume effects on the in-vivo MRI images. A total of 55 ROIs: 29 GM and 26 WM (one patient

140 had no WM ROIs as the resection did not include sufficient tissue), were segmented on histology

141 slices.

142

To ensure that equivalent ROIs were analyzed in each modality, we employed non-rigid 144 image registration to map the H\&E defined histology ROIs to the IHC slides and the in-vivo 145 MRI. Non-rigid image registration was performed between the in-vivo MRI and histology 146 images, using an ex-vivo MRI scan of the specimen as an intermediate reference image to 147 effectively split the registration in two steps. After surgical resection, each specimen was 148 oriented by the operating neurosurgeon, photographed and transported on ice to the imaging lab 149 for ex-vivo scanning, which was performed after overnight fixation in 10\% formalin. Each 150 specimen was wrapped in gauze for stabilization, transferred to suitably-sized containers for 151 imaging, and immersed in a fluorine-based fluid 'Christo-lube MCG 1046' (Lubrication 152 Technology, Inc) prior to imaging to avoid susceptibility artifacts at the tissue boundaries. The 153 specimen scanning was performed on the same 3 T MR scanner employed for patient imaging, 154 using a 6 channel coil designed to image the carotid artery. The sequences used for images that 155 are part of the registration pipeline are described in detail below. The T2-weighted images were 156 acquired with the fast imaging employing steady state acquisition (FIESTA) sequence (TR = $1578.17 \mathrm{~ms}, \mathrm{TE}=4.08 \mathrm{~ms}$, flip angle $=40^{\circ}, \mathrm{N}=2$, matrix $=200 \times 200$, slice thickness $=0.4, \mathrm{FOV}=$ $15870 \mathrm{~mm}$ ) with a resolution of $0.35 \times 0.35 \times 0.4 \mathrm{~mm}$. For cases where overnight imaging was 159 feasible and not disruptive to the clinical workflow $(\mathrm{N}=4)$, scanning was performed on a 9.4T 160 small bore Agilant MR magnet (Agilant, Santa Clara, CA, U.S.A) for improved image resolution 
161 and signal-to-noise ratio (SNR), as an alternative to the $3 \mathrm{~T}$ scan. The specimens were scanned 162 with an in-house developed coil for a total time of sixteen hours. For this protocol, images were 163 acquired with the TrueFisp sequence $\left(\mathrm{TR}=7.6 \mathrm{~ms}\right.$, TE $=3.8 \mathrm{~ms}$, flip angle $\left.=30^{\circ}\right)$ with an 164 isotropic resolution of $0.2 \mathrm{~mm}$ and a FOV of $(50 \times 26 \times 44)$ voxels. using a combination of image-based and landmark-based 3D deformable registration. The imagebased registration made use of a B-spline deformation field and a normalized mutual information (NMI) cost-function ${ }^{26}$, while the landmark registration relied on Gaussian radial basis functions

17127 . Validation of our registration protocol was achieved by computing target registration error 172 (TRE) based on manually-identified corresponding intrinsic anatomical landmarks, 173 demonstrating registration errors of $0.98 \pm 0.60 \mathrm{~mm}$ and $1.35 \pm 0.11 \mathrm{~mm}$ for histology to ex174 vivo and ex-vivo to in-vivo registrations respectively ${ }^{24}$. The IHC slides (NeuN and GFAP) were 175 linearly co-registered to the H\&E slides using downsampled grayscale images of each slide, with 176 registration accuracy better than $0.5 \mathrm{~mm}^{25}$. To avoid oblique resampling of the anisotropic 177 histology images, for the purposes of visualization and analysis, the in-vivo and ex-vivo images 178 were ultimately transformed to the space of the 3D reconstructed histology, Hist $3 D$, where the 179 reconstructed coronal histology slides are stacked parallel to the anterior-posterior axis. Figure 3 180 illustrates the four different spaces of MRI and histology, and registration results to bring both 181 modalities in alignment. All in-vivo quantitative maps (T1, T2, FA, MD, AD and RD) were 182 warped to the Hist3D space using the resultant deformation fields. Similarly, the histology ROIs 183 were mapped to the IHC slides and the intermediate space, and underwent a final step of manual 
184 correction, if needed, to account for potential registration errors and to circumvent partial volume 185 effects. ROIs transformed to in-vivo MRI space were used to obtain estimates of the mean MRI 186 parameter $\{\mathrm{T} 1, \mathrm{~T} 2, \mathrm{FA}, \mathrm{MD}, \mathrm{AD}$, and $\mathrm{RD}\}$ at each location in the plane corresponding to the 187 histology slides.

190 To assess the Gaussianity of the distribution of MRI samples, we employed the D'agostino \& 191 Pearson omnibus normality test. Linear mixed effects with random effects were employed to test 192 for relationships between MRI parameters and histological features. For these analyses the 193 histological features (stain field fraction, neuron size and neuron counts) were entered as 194 dependant variables and MRI parameters (T1, T2, FA, MD) from patients and slices as the 195 independent variables, whereas variables for both patient and slice were entered as repeated 196 measures. In addition, a random effect for subject was included to account for lower variance of 197 MRI parameters within a single (across slices) subject as compared to between subjects. For 198 fixed effects, we first fitted a model that included all MRI parameters as explanatory variables. 199 We then used a backward elimination procedure to retain significant variables only. We 200 employed Wald statistics for covariance structure selection. In addition, we assessed the 201 correlations between each of the above variables as well as seizure frequency, age at the time of 202 the last consultation prior to surgery, age of seizure onset, duration of epilepsy, and side of 203 onset/resection. We also looked at correlations between each MRI parameter and every other 204 MRI parameter, as well as those between histological features. We corrected for multiple 205 comparisons in our correlation analysis with family-wise error rate (FWER) control using 206 permutation tests ${ }^{28}$, and the presented $p$-values are adjusted for family-wise error. 
209 histology, we also employed linear mixed models between MRI parameters from each WM ROI

210 with histology features from its neighbouring GM ROI. Statistical analyses were performed in

211 IBM SPSS statistics 20 (IBM, Armonk, NY). To test whether combining multiple MRI

212 parameters leads to better prediction of histological features, multi-parametric models were

213 compared against simpler univariate models using likelihood ratio tests.

214

$215 \quad$ Results

216 MRI parameters-Histology features correlation

217 The registration protocol enabled us to determine precise correspondences between MR and

218 histology slices, and hence parameters from each slice were not averaged per patient and were

219 instead employed as unique data points in the analysis. $P$-values from the linear mixed effects

220 model analysis for the following histological features: neuron density, density (big neurons),

221 density (small neurons), NeuN field fraction, are summarized in Table 2. T1 was found to be a

222 significant predictor of total neuronal density in GM (Figure 4), as well as NeuN field fraction in

223 the GM. Moreover, when assessing different sub-types of neurons, T1 and FA were both found

224 to be predictors of neuronal density of large-caliber neurons (pyramidal cells) in the GM.

225 Furthermore, only T1 was to be a predictor of small-caliber neurons (granular cells) in the GM.

226 There were no significant associations between the GFAP field fraction and any MRI parameter

227 in either GM or WM. Similarly, no significant associations were seen between histology and

228 MRI parameters in the white matter. 
231 To test whether combining multiple MRI parameters leads to better prediction of histological

232 features, multi-parametric mixed effects models were compared against nested univariate models

233 using likelihood ratio tests and the chi-squared distribution. Multiple linear regression analysis

234 demonstrated that combining T1 and FA values predicted GM neuronal density of large-caliber 235 neurons with a better fit than T1 or FA on their own ( -2 log likelihood difference: 12.06, p $236<0.001)$. Other multi-parametric combinations however failed to demonstrate similar predictive 237 improvements. Figure 5 plots the samples in the space spanned by $\mathrm{T} 1$ and FA, revealing that 238 combining both parameters provides better discrimination of density of large neurons in 239 neocortical GM. Each dot in this plot refers to a gray matter ROI on a histology slide, with 240 representative dots being labeled with patient IDs from Table 1. It is clear from the figure that 241 low and high neuron densities are not well separated when using T1 or FA (see projections on 242 horizontal and vertical axes), but in the two-dimensional space the data are more clearly 243 separable (demonstrated by the dashed line), suggesting that multivariate or multi-parametric 244 analysis would be more beneficial in predicting or classifying pathology in-vivo. Since the 245 presented ROIs are extracted from sparsely sectioned histology slices (4 mm apart), this figure 246 highlights the potential of imaging parameters in detecting local pathology within the neocortex.

249 Correlations with clinical variables revealed significant positive correlations between neuronal 250 density and age $\left(r_{\mathrm{s}}=0.726, \mathrm{p}_{\mathrm{fwe}}=0.021\right)$. Finally there were significant correlations with side of 251 seizure onset, with left TLE patients exhibiting increased GM T1 ( $\mathrm{r}_{\mathrm{s}}=0.671$, $\left.\mathrm{p}_{\mathrm{fwe}}=0.042\right)$. 
252 There were no correlations between clinical variables with MRI parameters and histological

253 features in the white matter.

255 MRI-MRI parameters correlation

256 Table 3 summarizes the correlations between all MRI parameters within both tissue types (GM 257 and WM). When assessing the relationships between diffusion and relaxometry parameters, there 258 was a negative correlation between T1 and FA in WM, as well as a positive correlation between 259 T1 values and MD in WM. When assessing the relationships between diffusion parameters (FA 260 vs. MD) and relaxation parameters (T1 vs. T2) no significant correlations were found after 261 multiple comparison correction. Figure 6 demonstrates the significant relationships between 262 diffusion and relaxometry MRI parameters.

263

264 Histology-Histology features correlation

265 We found a positive correlation between neuronal density and NeuN field fraction in GM $\left(\mathrm{r}_{\mathrm{s}}=\right.$ $2660.929, p=4.0 \times 10^{-09}$ ), as shown in Figure 6. Similarly, neuronal density was positively correlated 267 with densities of both large and small neurons in GM when analyzed separately $\left(\mathrm{r}_{\mathrm{s}}=0.93, \mathrm{p}=\right.$ $2681.4 \times 10^{-09}$ and $\left.r_{s}=0.95, p=1.4 \times 10^{-10}\right)$. In addition, a slightly higher correlation was detected 269 between NeuN field fraction and density of larger neurons in GM $\left(\mathrm{r}_{\mathrm{s}}=0.96, \mathrm{p}=1.5 \times 10^{-11}\right)$, than 270 with density of smaller neurons in GM $\left(r_{s}=0.83, p=1.8 \times 10^{-05}\right)$. Finally, the association between

271 both measurements of densities proved positively correlated as well in $\mathrm{GM}\left(\mathrm{r}_{\mathrm{s}}=0.80, \mathrm{p}=\right.$ $\left.2721.1 \times 10^{-04}\right)$. 


\section{Neurobiological interpretations and considerations}

276 A significant finding of this work was the negative association between T1 values and neuronal 277 integrity measures (NeuN field fraction, neuronal density) in the gray matter. T1 relaxation is

278 related to many factors in the tissue, including macromolecular integrity and the relationship 279 between free and bound water. Neuronal loss will likely result in an overall loss of 280 macromolecules and an increase in the extra-cellular space (thus increased amount of extra281 cellular water and decreased amount of intra-cellular water), all of which would act to increase $282 \mathrm{~T} 1{ }^{29}$. A similar relationship between ex-vivo GM T1 values and neuronal density has been 283 described in patients with multiple sclerosis ${ }^{30}$. Our study is the first to observe this relationship 284 with in-vivo quantitative T1 mapping and in temporal lobe resections. Eriksson et al. (2007) ${ }^{16}$ 285 found correlations between T2 and gray matter NeuN field fraction, employing a dual-echo fast 286 FLAIR T2 (FFT2) mapping at $1.5 \mathrm{~T}$ with a $5 \mathrm{~mm}$ slice thickness. One possible explanation for 287 why we did not observe this trend with our T2 maps is differences in the mapping protocols; our 288 protocol at 3T, had significantly thinner slices, and did not use a fluid-attenuated inversion 289 recovery (FLAIR) sequence. We plan to compare the relationship between our T1 and T2 maps and FLAIR sequences in future work to better understand the effectiveness of each technique in assessing pathology. neurons in the cortical gray matter. While this seems counterintuitive to our expectations in white matter, where a decrease in FA is usually associated with pathology, the cyto- and myelo296 architecture in the cortex is considerably different from that in the white matter. Moreover, an 297 increase in anisotropy was previously reported ${ }^{31}$ within the dentate gyrus in an animal model of 
298 seizing rats, as compared to naive controls. Diffusion anisotropy is low and not typically 299 examined in the cortical gray matter, with some exceptions ${ }^{32}$. However high-resolution diffusion 300 studies on post-mortem brains have shown that the fibre configuration can be complex, with both 301 fibres parallel and perpendicular to the cortical surface observed, along with areas of fibre 302 crossings ${ }^{33}$. In a region of low anisotropy due to fibre-crossing, such as the cortex, selective loss 303 of one type of fibres would lead to an increase in anisotropy (i.e. a shift to a simpler fibre 304 configuration). This phenomenon has been observed previously in a region of white matter fibre305 crossing, where Douaud et al. $(2011)^{34}$ demonstrated an increase of FA could be explained by a 306 relative preservation of motor-related projection fibres crossing the association fibres of the 307 superior longitudinal fasciculus in subjects with mild cognitive impairment. Thus, the increase in

308 FA we observed, coinciding with a loss of only large-calibre neurons, could be explained by the 309 selective loss of fibres running either parallel or perpendicular to the cortical surface), as 310 depicted in the simplified schematic representation in Figure 7. Given the limitations of in-vivo 311 DTI data we cannot precisely assess the nature of the architectural changes related to FA, 312 however we hope to explore these issues further using high-resolution ex-vivo DTI of the 313 resected specimens.

In the white matter, increases in $\mathrm{T} 1$ were positively correlated with MD and negatively 316 with FA. This agrees with previous studies that have also demonstrated reduced FA and 317 increased MD in the ipsilateral white matter in TLE ${ }^{35-39}$. These changes may be due to 318 degeneration of axons, reduced packing, or demyelination ${ }^{40}$ which may facilitate isotropic 319 diffusion and accumulation of free water in the extracellular space, which would lengthen T1 as 320 well. A similar trend of prolonged T1 times and decreased FA was reported in white matter 
321 hyperintense regions of Alzheimer's patients ${ }^{41}$, where they showed that increased T1 reflected a 322 range of pathological findings including axon and myelin loss and microglial activation, whereas 323 the strongest predictor of decreased FA was axonal loss. In addition to affecting relaxation and 324 diffusion parameters, reactive gliosis has also been previously associated with neuronal loss ${ }^{42}$, 325 however we did not observe any significant correlations with GFAP IHC in either GM or WM.

326

327

328 329 330 331 332 338 dominance ${ }^{47}$.

previously in a healthy aging population ${ }^{43}$, and was attributed to atrophy (volume loss) without accompanied neuronal loss. This has potential implications on the detection of neuronal integrity, since if age-related atrophy (density increases) and neuron loss (density decreases) occur simultaneously, there may be no net change in density, and thus no change in MRI signal. Finally, we found significant differences in left-onset TLE patients, which had increased T1 and decreased FA in the gray matter. Asymmetry has also been found in other recent DTI studies ${ }^{44}$, 45 with left-onset patients having more significant and widespread abnormalities and greater hippocampal atrophy ${ }^{46}$, and have been speculated to be due to the greater vulnerability to early injury and the progressive effect of seizures on the left hemisphere. These asymmetric structural differences could also be related to the inherent functional lateralization, including language

\section{Benefit of registration-based correlation}

341 Many studies correlating MRI and histology have been performed without the use of 342 computational methods for 3D image registration, relying instead on visual matching of ROIs. 343 However, this is difficult in cases where the visibility or boundaries of the lesion in MRI and 
344 histology differ and where no definitive lesion is apparent (as in paradoxical TLE). Another 345 drawback of visual matching is that it becomes more challenging to find corresponding slices 346 when there are 3D deformations present, as the anatomy in a histology slice may not be fully 347 present in a single MRI slice, even if obliquely resampled. If no registration is employed and the 348 tissue is subjected to non-rigid deformations, the samples from both modalities may represent 349 different parts of the same anatomical region, which could potentially lead to abnormal sub350 regions of one modality being correlated with normal elements of the other. When image 351 registration is employed, the degree of mismatch between regions of both modalities becomes 352 dependant on the registration error. For example, an image registration error between in-vivo 353 MRI and histology of $1 \mathrm{~mm}$ would produce an overlap of $70 \%$ between two regions of interests 354 with a volume of $140 \mathrm{~mm}^{3}$ on each modalities, (roughly the size of a very small FCD) ${ }^{48}$.

\section{Limitations and future work}

357 The current study is limited to the investigation of neuronal integrity and gliosis through field 358 fractions and measurements of neuronal size and density. Since focal neuronal loss and gliosis 359 are thought to be related to epileptogenicity, correlation of these measures with MRI is an 360 important step in validating quantitative imaging techniques. Additional insight might also be 361 gained through the use of myelin-specific stains (Luxol fast blue, or myelin basic protein), since 362 their relationship with both $\mathrm{T} 1{ }^{49}$ and $\mathrm{T} 2{ }^{30}$ has been previously demonstrated. Another limitation 363 of this work is the lack of normative control data for histology. Several post-mortem control neocortical specimens were acquired for histological analysis; however the staining ability of 365 NEUN degrades with time after formalin fixation ${ }^{50}$. Moreover, it is logistically very difficult to 366 obtain ethics approval for acquiring and handling fresh (unfixed) brain control specimens. We 
367 plan to address these issues in future studies. The lack of control non-epileptic specimens makes

368 it difficult to validate that the observed pathological changes directly relate to seizure generation, 369 and hence the presented findings should be considered preliminary.

In addition to histopathology, correlation with electrophysiology obtained with

372 intracranial EEG (iEEG) could be used to further validate these techniques and better understand

373 the relationship with epileptogenicity, imaging, and histology. However, there are some issues

374 with using iEEG as a gold-standard for validating imaging methods, since localization is limited

375 to placement of the electrodes and abnormal iEEG may not actually have an altered structural

376 substrate that can be detected. For these reasons, it may still be more beneficial to investigate the

377 histopathological correlates instead of iEEG, specifically of cortical dysplasia, which often go

378 undetected and have a higher risk for seizure recurrence ${ }^{51}$. We intend as well to correlate our

379 imaging findings (specifically abnormalities found on T1 and FA maps) with long-term seizure

380 outcomes, and investigate whether the absence of such lesions provides more favourable

381 outcomes for MRI-negative patients, as well as patients undergoing surgery due to hippocampal

382 sclerosis. Future work should as well investigate whether these neocortical abnormalities are

383 related to the epileptogenicity in those patients, possibly though correlation of the imaging

384 abnormalities with depth electrodes recordings on truly MRI-negative patients (those without

385 any identifiable lesions whether in the hippocampus or neocortex). Better quantification and

386 characterization of these lesions in histology, based on neuronal- and laminar-centric analysis,

387 could be used to improve detection and precise delineation with MRI, and could improve

388 surgical outcomes through more complete resection of the underlying pathology ${ }^{52}$. Our future 


\section{Conclusion}

393

394 395 degeneration in neurodegenerative diseases as Alzheimer's.

In conclusion, we have demonstrated that alterations of in-vivo $\mathrm{T} 1$ and FA, in the temporal lobe cortex and white matter, are predictive of neuronal integrity (density and size) that serve to delineate an epileptogenic lesion. Our study is the first to quantitatively assess the relationship between MRI and histopathological features using correspondences based on image registration in focal epilepsy, and to relate in-vivo T1 and FA values to the proportion of neurons, specifically large-caliber neurons, in the neocortical gray matter. Our registration and correlation pipeline allows for a quantitative assessment of the pathological correlates of MRI by bringing information from both modalities, and the potential prediction of pathology from in-vivo MRI. This study suggests that quantitative MRI sequences, specifically multi-parameter T1 mapping and DTI, may have a role in routine clinical practice for pre-operative evaluation of focal epilepsy and motivates further investigation in this area. These in-vivo quantitative maps can be extended as well to identify gray matter lesions in multiple sclerosis or be used as a marker for

407
microscopy to accurately quantify and characterize these lesions for correlation with MRI. 


\section{Acknowledgments}

415 The authors would like to thank Cathie Crukley, Robert Mayer and Catherine Currie for their 416 assistance and support throughout the study.

417

418 This project is funded by the Canadian Institute of Health Research (CIHR) grant MOP 184807

419 and Canada Foundation for Innovation (CFI) grant 20994. MG is supported by the NSERC

420 Create Grant CAMI award at Western University. AK is supported by a post-doctoral fellowship 421 from the Canadian Institute of Health Research (CIHR).

422

423

424

425

426

427

428

429

430

431

432

433

434 
$437 \quad \underline{\text { References }}$

438 1. Engel J. Etiology as a risk factor for medically refractory epilepsy: A case for early surgical 439 intervention. Neurology 1998;51:1243-1244.

440 2. Engel J. Mesial temporal lobe epilepsy: What have we learned? Neuroscientist 2001;7:340441352.

442 3. Wiebe S, Blume WT, Girvin J P, Eliasziw M. A randomized, controlled trial of surgery for 443 temporal-lobe epilepsy. New England Journal of Medicine 2001;345:311-318.

444 4. de Tisi J, Bell GS, Peacock JL, McEvoy AW, Harkness WF, Sander JW, et al. The long-term 445 outcome of adult epilepsy surgery, patterns of seizure remission, and relapse: A cohort study. $446 \quad$ The Lancet 2011;378:1388-1395.

447 5. Najm I, Jehi L, Palmini A, Gonzalez-Martinez J, Paglioli E, Bingaman W. Temporal patterns 448 and mechanisms of epilepsy surgery failure. Epilepsia 2013;54:772-782.

449 6. Blume WT, Ganapathy GR, Munoz D, Lee DH. Indices of resective surgery effectiveness for 450 intractable nonlesional focal epilepsy. Epilepsia 2004;45:46-53.

451 7. Jeha L, Najm I, Bingaman W, Khandwala F, Widdess-Walsh P, Morris H, et al. Predictors of 452 outcome after temporal lobectomy for the treatment of intractable epilepsy. Neurology $453 \quad 2006 ; 66: 1938-1940$.

454 8. Cohen-Gadol AA, Bradley CC, Williamson A, Kim JH, Westerveld M, Duckrow RB, et al. 455 Normal magnetic resonance imaging and medial temporal lobe epilepsy: The clinical 456 syndrome of paradoxical temporal lobe epilepsy. J Neurosurg 2005;102:902-909. 
457 9. Sylaja P, Radhakrishnan K, Kesavadas C, Sarma P. Seizure outcome after anterior temporal 458 lobectomy and its predictors in patients with apparent temporal lobe epilepsy and normal $459 \quad$ MRI. Epilepsia 2004;45:803-808.

460 10. Lee SK, Lee SY, Kim K, Hong K, Lee D, Chung C. Surgical outcome and prognostic factors 461 of cryptogenic neocortical epilepsy. Ann Neurol 2005;58: 525-532.

462 11. Widdess-Walsh P, Diehl B, Najm I. Neuroimaging of focal cortical dysplasia. Journal of $463 \quad$ Neuroimaging 2006;16:185-196.

464 12. Bernasconi A, Bernasconi N, Caramanos Z, et al. T2 relaxometry can lateralize mesial 465 temporal lobe epilepsy in patients with normal MRI. Neuroimage 2000;12:739-746.

13. Bernasconi N, Duchesne S, Janke A, Lerch J, Collins D, Bernasconi A. Whole-brain voxelbased statistical analysis of gray matter and white matter in temporal lobe epilepsy. Neuroimage 2004;23:717-723.

14. Bernhardt B, Worsley K, Kim H, Evans A, Bernasconi A, Bernasconi N. Longitudinal and cross-sectional analysis of atrophy in pharmacoresistant temporal lobe epilepsy. Neurology 2009;72:1747-1754.

15. Garbelli R, Milesi G, Medici V, Villani F, Didato G, Deleo F, et al. Blurring in patients with temporal lobe epilepsy: Clinical, high-field imaging and ultrastructural study. Brain 2012;135:2337-2349.

16. Eriksson S, Free S, Thom M, Martinian L, Symms M, Salmenpera T, et al. Correlation of quantitative MRI and neuropathology in epilepsy surgical resection specimens-T2 correlates with neuronal tissue in gray matter. Neuroimage 2007;37:48-55. 
478 17. Eriksson SH, Free SL, Thom M, Symms MR, Martinian L, Duncan JS, et al. Quantitative gray matter histological measures do not correlate with gray matter probability values from in vivo MRI in the temporal lobe. J Neurosci Methods 2009;181:111-118.

18. Lockwood-Estrin G, Thom M, Focke NK, Symms MR, Martinian L, Sisodiya SM, et al.

482 Correlating 3T MRI and histopathology in patients undergoing epilepsy surgery. J Neurosci Methods 2012;205:182-189.

19. Deoni SC. High-resolution T1 mapping of the brain at 3T with driven equilibrium single 485 pulse observation of T1 with high-speed incorporation of RF field inhomogeneities 486 (DESPOT1-HIFI). J Magn Reson Imaging 2007;26:1106-1111.

20. Deoni SC. Transverse relaxation time (T2) mapping in the brain with off-resonance correction using phase-cycled steady-state free precession imaging. J Magn Reson Imaging

21. Ruifrok AC and Johnston DA. Quantification of histochemical staining by color deconvolution. Anal Quant Cytol Histol. 2001;23(4):291-299.

22. Soille P. Morphological Image Analysis: Principles and Applications. Springer-Verlag New 493 York, Inc; 2003.

494 23. Yushkevich PA, Piven J, Hazlett HC, Smith RG, Ho S, Gee JC, et al. User-guided 3D active 495 contour segmentation of anatomical structures: Significantly improved efficiency and reliability. Neuroimage 2006;31:1116-1128.

497 24. Goubran M, Khan AR, Crukley C, Buchanan S, Santyr B, deRibaupierre S, Peters TM. 498 Robust registration of sparsely sectioned histology to ex-vivo MRI of temporal lobe resections. Proc. SPIE 8314, Medical Imaging 2012;8314:83141V. 
500

501

502

503

504

505

506

507

508

509

510

511

512

513

514

515

516

517

518

519

520

521

25. Goubran M, Crukley C, de Ribaupierre S, Peters TM, Khan AR. Image registration of exvivo MRI to sparsely sectioned histology of hippocampal and neocortical temporal lobe specimens. Neuroimage 2013;83:770-781.

26. Modat M, Ridgway GR, Taylor ZA, Lehmann M, Barnes J, Hawkes DJ, et al. Fast free-form deformation using graphics processing units. Comput Methods Programs Biomed 2010;98:278-284.

27. Pinter C, Lasso A, Wang A, Jaffray D, Fichtinger G. SlicerRT: Radiation therapy research toolkit for 3D Slicer. Medical physics 2012;39:6332-6338.

28. Groppe DM, Urbach TP, Kutas M. Mass univariate analysis of event-related brain potentials/fields I: A critical tutorial review. Psychophysiology 2011;48:1711-1725.

29. Jurcoane A, Wagner M, Schmidt C, Mayer C, Gracien R, Hirschmann M, et al. Within-lesion differences in quantitative MRI parameters predict contrast enhancement in multiple sclerosis. J Magn Reson Imaging 2013;38:1454-1461.

30. Schmierer K, Parkes HG, So P-, An SF, Brandner S, Ordidge RJ, et al. High field (9.4 tesla) magnetic resonance imaging of cortical gray matter lesions in multiple sclerosis. Brain 2010;133:858-867.

31. Parekh MB, Carney PR, Sepulveda H, Norman W, King M, Mareci TH. Early MR diffusion 17 and relaxation changes in the parahippocampal gyrus precede the onset of spontaneous seizures in an animal model of chronic limbic epilepsy. Expr Neurol 2010;224:258-270.

32. Kang DH, Jo HJ, Jung WH, Kim SH, Jung YH, Choi CH, et al. The effect of meditation on brain structure: cortical thickness mapping and diffusion tensor imaging. Soc Cogn Affect Neurosci 2013;8:27-33. 
522 33. Leuze CW, Anwander A, Bazin PL, Dhital B, Stüber C, Reimann K, et al. Layer-specific intracortical connectivity revealed with diffusion MRI. Cereb Cortex 2014;24:328-339.

524 34. Douaud G, Jbabdi S, Behrens TEJ, Menje RA, Gass A, Monsch AU, et al. DTI measures in 525 crossing-fibre areas: Increased diffusion anisotropy reveals early white matter alteration in 526 MCI and mild Alzheimer's disease. Neuroimage 2011;55:880-890.

527 35. Rugg-Gunn FJ, Eriksson SH, Symms MR, Barker GJ, Thom M, Harkness W, et al. Diffusion 528 sclerosis. Neuroimage 2008;40:728-737.

37. Salmenpera TM, Simister RJ, Bartlett P, Symms MR, Boulby PA, Free SL, et al. Highresolution diffusion tensor imaging of the hippocampus in temporal lobe epilepsy. Epilepsy

36. Focke NK, Yogarajah M, Bonelli SB, Bartlett PA, Symms MR, Duncan JS. Voxel-based diffusion tensor imaging in patients with mesial temporal lobe epilepsy and hippocampal Res 2006;71:102-106.

38. Thivard L, Lehéricy S, Krainik A, Adam C, Dormont D, Chiras J, et al. Diffusion tensor imaging in medial temporal lobe epilepsy with hippocampal sclerosis. Neuroimage 2005;28:682-690.

39. Khan AR, Goubran M, de Ribaupierre S, Hammond RR, Burneo JG, Parrent AG, Peter TM. Quantitative relaxometry and diffusion MRI for laterization in MTS and non-MTS temporal lobe epilepsy. Epilepsy Research 2014;108:506-516.

40. Gross DW. Diffusion tensor imaging in temporal lobe epilepsy. Epilepsia 2011;52:32-34.

41. Gouw AA, Seewann A, Vrenken H, van der Flier WM, Rozemuller JM, Barkhof F, et al. Heterogeneity of white matter hyperintensities in alzheimer's disease: Post-mortem quantitative MRI and neuropathology. Brain 2008;131:3286-3298. 
42. Block ML, Zecca L, Hong J. Microglia-mediated neurotoxicity: Uncovering the molecular mechanisms. Nature Reviews Neuroscience 2007;8:57-69.

43. Freeman SH, Kandel R, Cruz L, Rozkalne A, Newell K, Frosch MP, Hedley-Whyte ET, et al. Preservation of neuronal number despite age-related cortical brain atrophy in elderly subjects without Alzheimer disease. J Neuropathol Exp Neurol 2008;67(12):1205-1212.

44. Shon Y, Kim Y, Koo B, Lee J, Kim HJ, Kim WJ, et al. Group-specific regional white matter abnormality revealed in diffusion tensor imaging of medial temporal lobe epilepsy without hippocampal sclerosis. Epilepsia 2010;51:529-535.

45. Kemmotsu N, Girard HM, Kucukboyaci N E, McEvoy LK, Hagler DJ, Dale AM, et al. Agerelated changes in the neurophysiology of language in adults: Relationship to regional cortical thinning and white matter microstructure. J Neurosci 2012;32:12204-12213.

46. Bonilha L, Rorden C, Halford, J J, Eckert M, Appenzeller S, Cendes F, et al. Asymmetrical extra-hippocampal gray matter loss related to hippocampal atrophy in patients with medial temporal lobe epilepsy. J Neurol Neurosurg Psychiatry 2007;78:286-294.

47. Barrick TR, Lawes IN, Mackay CE, Clark CA. White matter pathway asymmetry underlies functional lateralization. Cereb Cortex 2007;17(3):591-598.

48. Besson P, Bernasconi N, Colliot O, Evans A, Bernasconi A. Surface-based texture and morphological analysis detects subtle cortical dysplasia. In: Medical Image Computing and Computer-Assisted Intervention--MICCAI 2008;2008:645-652.

49. Mottershead J, Schmierer K, Clemence M, Thornton J, Scaravilli F, Barker G, et al. High field MRI correlates of myelin content and axonal density in multiple sclerosis. J Neurol 2003;250:1293-1301. 
567 50. Lyck L, Dalmau I, Chemnitz J, Finsen B, Daa Schroder H. Immunohistochemical Markers 568 for Quantitative Studies of Neurons and Glia in Human Neocortex. Journal of Histochemistry 569 \& Cytochemistry 2008;56:201-221.

570 51. Palmini A, Gambardella A, Andermann F, Dubeau F, da Costa JC, Olivier A, et al. Operative 571 strategies for patients with cortical dysplastic lesions and intractable epilepsy. Epilepsia $572 \quad 1994 ; 35: 57-71$.

573 52. Fauser S, Schulze-Bonhage A, Honegger J, Carmona H, Huppertz HJ, Pantazis G, et al. 574 Focal cortical dysplasias: surgical outcome in 67 patients in relation to histological subtypes 575 and dual pathology. Brain 2004;127:2406-2418. 
Table 1. Patient demographics and clinical information including age, gender, onset age, seizure origin as well as clinical MRI and pathology findings for our patient cohort. MTS = mesial temporal sclerosis, MAA= minor architectural abnormalities, Neo. Path. = Neocortical Pathology, Hp. Path. $=$ Hippocampal Pathology $\dagger$ Previous resection of left temporal lobe tumour (DNET), * not enough tissue to make diagnosis of MTS

\begin{tabular}{|c|c|c|c|c|c|c|c|c|c|c|}
\hline Patient & Gender & Age & $\begin{array}{c}\text { Age } \\
\text { of } \\
\text { Onset }\end{array}$ & $\begin{array}{c}\mathrm{Sz} \\
\text { Origin }\end{array}$ & $\begin{array}{c}\text { Sz } \\
\text { Freq. } \\
\text { /month }\end{array}$ & MRI & Neo. Path. & Hp. Path. & $\begin{array}{c}\text { Engel } \\
\text { outcome }\end{array}$ & $\begin{array}{c}\text { Yrs } \\
\text { since } \\
\text { surger: }\end{array}$ \\
\hline 1 & $\mathrm{~F}$ & 25 & 17 & $\mathrm{~L}$ & 2 & Normal † & $\begin{array}{c}\text { Gliosis, Ki67- } \\
\text { positive cells } \\
\text { in } \mathrm{WM} \dagger\end{array}$ & Gliosis & 3 & 2.0 \\
\hline 2 & M & 20 & 3 & $\mathrm{~L}$ & 16 & MTS & Gliosis, MAA & MTS & 2 & 2.1 \\
\hline 3 & M & 18 & 14 & $\mathrm{R}$ & 32 & $\begin{array}{c}\text { Possible } \\
\text { MTS }\end{array}$ & Gliosis, MAA & Gliosis* & 1 & 2.1 \\
\hline 4 & $\mathrm{~F}$ & 48 & 36 & $\mathrm{~L}$ & 28 & MTS & Gliosis & MTS & 1 & 1.7 \\
\hline 5 & $\mathrm{~F}$ & 50 & 47 & $\mathrm{~L}$ & 20 & $\begin{array}{l}\text { GM/WM } \\
\text { blurring }\end{array}$ & Gliosis & Gliosis* & 1 & 1.6 \\
\hline 6 & M & 31 & 28 & $\mathrm{R}$ & 2 & Normal & $\begin{array}{c}\text { Mild gliosis, } \\
\text { MAA }\end{array}$ & $\begin{array}{c}\text { Negligible } \\
\text { gliosis }\end{array}$ & 1 & 1.3 \\
\hline 7 & $\mathrm{~F}$ & 32 & 19 & $\mathrm{~L}$ & 2 & MTS & Gliosis & MTS & 1 & 1.2 \\
\hline 8 & $\mathrm{~F}$ & 43 & 3 & $\mathrm{R}$ & 4 & MTS & Gliosis & MTS & 2 & 1.4 \\
\hline 9 & $\mathrm{~F}$ & 26 & 19 & $\mathrm{R}$ & 12 & $\begin{array}{l}\text { Cortical } \\
\text { tubers }\end{array}$ & $\begin{array}{c}\text { Dysplastic } \\
\text { lesion, cortical } \\
\text { tuber }\end{array}$ & Gliosis & 2 & 2.5 \\
\hline 10 & M & 34 & 15 & $\mathrm{~L}$ & 2 & MTS & $\begin{array}{c}\text { Gliosis, focal } \\
\text { MAA }\end{array}$ & MTS & 3 & 1.2 \\
\hline 11 & $\mathrm{~F}$ & 40 & 7 & $\mathrm{R}$ & 20 & $\begin{array}{c}\text { MTS, } \\
\text { Porencephaly }\end{array}$ & Gliosis, MAA & MTS & 2 & 2.5 \\
\hline 12 & $\mathrm{~F}$ & 56 & 15 & $\mathrm{R}$ & 8 & Normal & $\begin{array}{c}\text { Gliosis, } \\
\text { arteriosclerosis }\end{array}$ & Gliosis* & 1 & 1 \\
\hline 13 & M & 23 & 18 & $\mathrm{~L}$ & 12 & Normal & $\begin{array}{c}\text { Gliosis, MAA, } \\
\text { possible FCD } \\
\text { type 1a }\end{array}$ & Gliosis & 1 & 1 \\
\hline
\end{tabular}


Table 2. Results of the linear mixed-effects models with random effects for subjects, showing $p$ values for variables with significant fixed effects.

\begin{tabular}{ccccc}
\hline $\begin{array}{c}\text { Grey } \\
\text { matter }\end{array}$ & Neuron density & $\begin{array}{c}\text { Neuron density } \\
\text { (large neurons) }\end{array}$ & $\begin{array}{c}\text { Neuron density } \\
\text { (small neurons) }\end{array}$ & $\begin{array}{c}\text { NEUN field } \\
\text { fraction }\end{array}$ \\
\hline T1 & 0.007 & 0.019 & 0.004 & 0.001 \\
\hline T2 & & & \\
\hline FA & 0.009 & & \\
\hline MD & & & \\
\hline
\end{tabular}

The minimum adequate model was obtained by backward selection removing the nonsignificant fixed effects. 
Table 3. Significance of Spearman Rho correlations between MR parameters. All $p$-values were corrected for family wise error.

\begin{tabular}{|c|c|c|c|c|}
\hline $\begin{array}{l}\text { Grey matter } \\
\text { parameters }\end{array}$ & $\mathbf{T 1}$ & $\mathbf{T} 2$ & FA & MD \\
\hline $\mathbf{T 1}$ & & $\begin{array}{l}\mathrm{r}=0.323 \\
\mathrm{P}=0.428\end{array}$ & $\begin{array}{l}\mathrm{r}=-0.207 \\
\mathrm{P}=0.515\end{array}$ & $\begin{array}{l}\mathrm{r}=0.129 \\
\mathrm{P}=0.843\end{array}$ \\
\hline $\mathbf{T} 2$ & & & $\begin{array}{l}\mathrm{r}=-0.316 \\
\mathrm{P}=0.463\end{array}$ & $\begin{array}{c}\mathrm{r}=-0.036 \\
\mathrm{P}=0.931\end{array}$ \\
\hline FA & & & & $\begin{array}{l}\mathrm{r}=-0.188 \\
\mathrm{P}=0.612\end{array}$ \\
\hline MD & & & & \\
\hline $\begin{array}{l}\text { White matter } \\
\text { parameters }\end{array}$ & $\mathbf{T 1}$ & $\mathbf{T} 2$ & FA & MD \\
\hline $\mathbf{T 1}$ & & $\begin{array}{l}r=0.464 \\
P=0.144\end{array}$ & $\begin{array}{c}r=-0.806 \\
\mathbf{P}=\mathbf{1 . 7 3 e - 0 4 *}\end{array}$ & $\begin{array}{l}\mathrm{r}=0.643 \\
\mathbf{P}=\mathbf{0 . 0 3 2}\end{array}$ \\
\hline $\mathbf{T} 2$ & & & $\begin{array}{c}r=-0.229 \\
P=0.639\end{array}$ & $\begin{array}{l}\mathrm{r}=0.381 \\
\mathrm{P}=0.241\end{array}$ \\
\hline FA & & & & $\begin{array}{l}\mathrm{r}=-0.527 \\
\mathrm{P}=0.147\end{array}$ \\
\hline MD & & & & \\
\hline
\end{tabular}




\section{Figure legends}

Figure 1. Histological processing and semi-quantitative features extraction, for both NeuN and GFAP IHC stains.

Figure 2. ROI placement and MRI parameters extraction. A) Gray matter and white ROI on 100um H\&E histology slice in histology native space. B) Registered ex-vivo MRI slice corresponding to the histology slice in Hist $3 D$ space. C) Warped ROIs overlaid on the registered and obliquely resampled T1 map in Hist $3 D$ space where MRI parameters extraction is performed. The registered histology slice is shown in the top left corner. D) Warped ROIs in the native in-vivo MRI space overlaid on three consecutive slices of the T1 map for illustration purposes.

Figure 3. Overview of our registration pipeline depicting registration results and the four different spaces of MRI and histology including the intermediate Hist $3 D$ space where reconstructed histology slices are stacked parallel to the A-P axis.

Figure 4. Relationships between quantitative MRI parameters (T1 and FA) and neuronal density in GM (Top left: Total neuronal density, Top right: Neuronal density for small-caliber neurons, Bottom: Neuronal density for large-caliber neurons).

Figure 5. Representation of T1-FA multi-parametric space, revealing that combining T1 and FA provides better discrimination of normal and abnormal neuron density in neocortical gray matter. Each dot in this plot refers to a gray matter ROI on a histology slide, with representative dots being labeled with patient IDs from Table 1. It is clear from the figure that low and high neuron densities are not well separated when using T1 or FA (see projections on horizontal and vertical axes), but in the two-dimensional space the data demonstrate are more clearly separable (demonstrated by the dashed line), suggesting that multivariate or multi-parametric analysis 
would be more beneficial in predicting or classifying pathology in-vivo. Since the presented ROIs are extracted from sparsely sectioned histology slices (4 $\mathrm{mm}$ apart), this figure highlights the potential of imaging parameters in detecting local pathology within the neocortex.

Figure 6. Significant relationships between diffusion and relaxometry MRI parameters, as well as the association between neuronal density and NeuN field fraction.

Figure 7. Summary of MRI parameters and histological features correlations in both tissues of the temporal lobe neocortex, along with possible neurobiological explanations for the highlighted relations. 


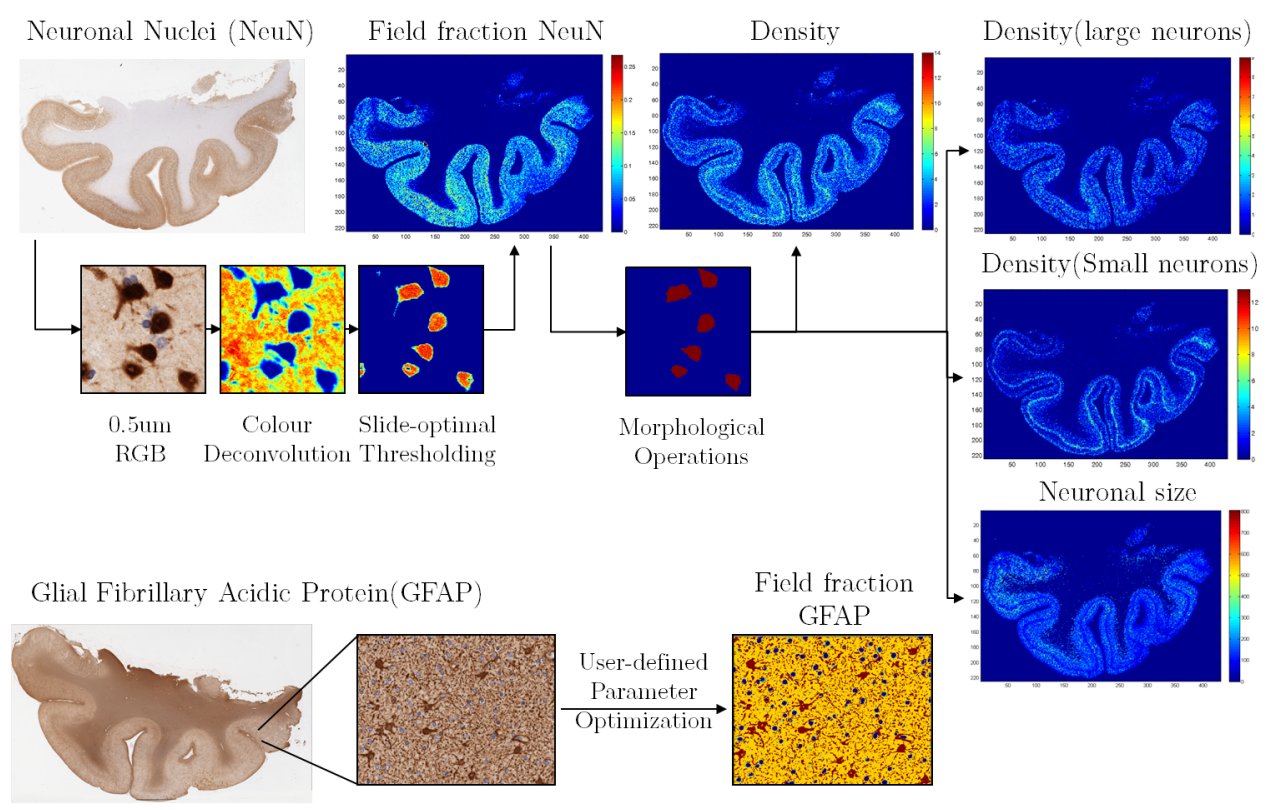




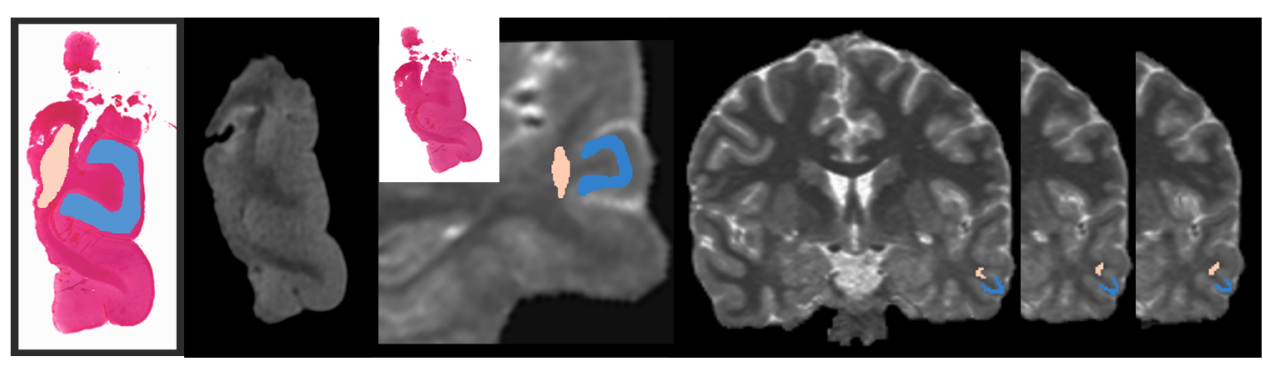

a)

b)

c)

d) 


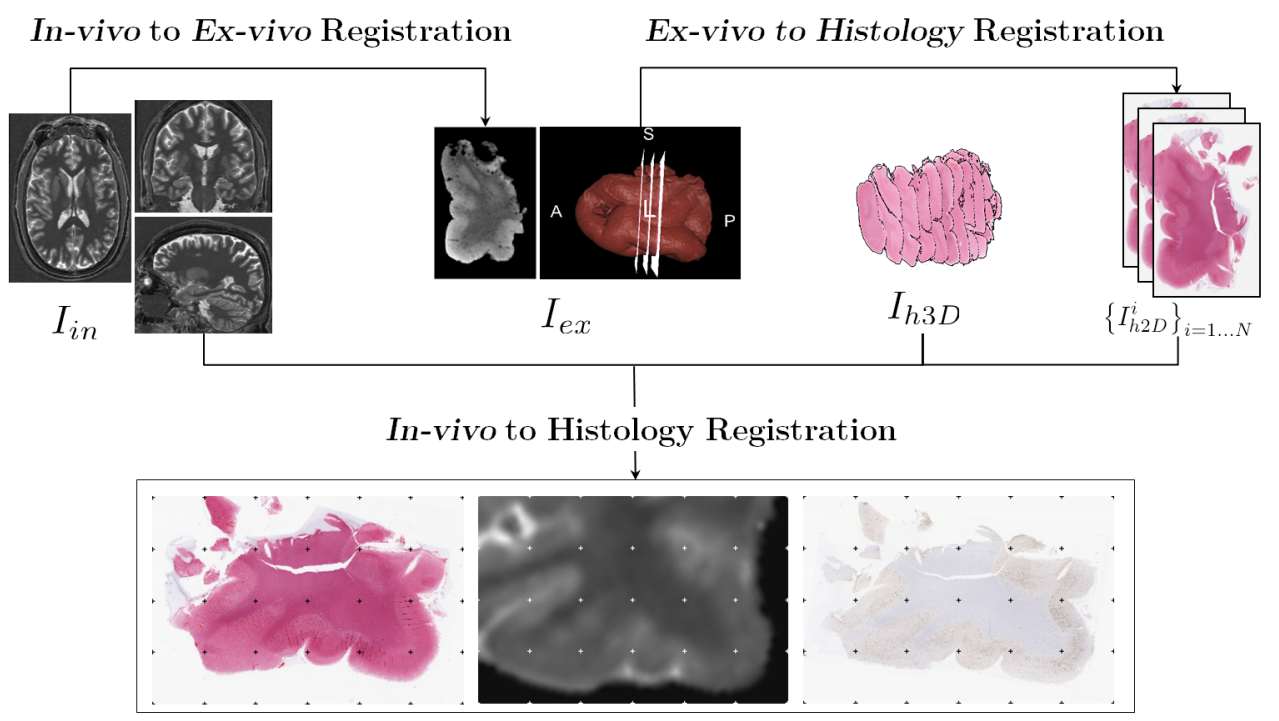



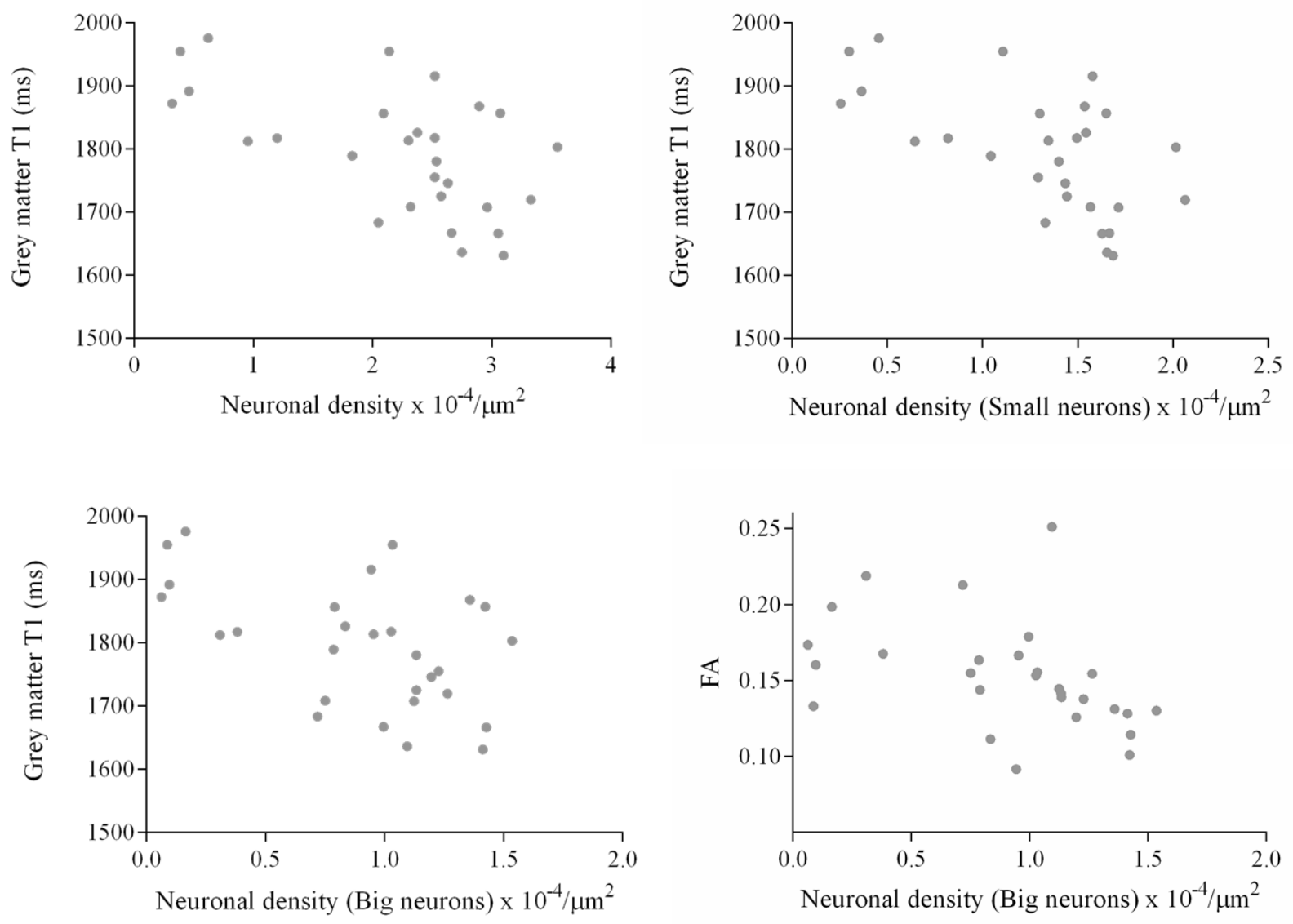


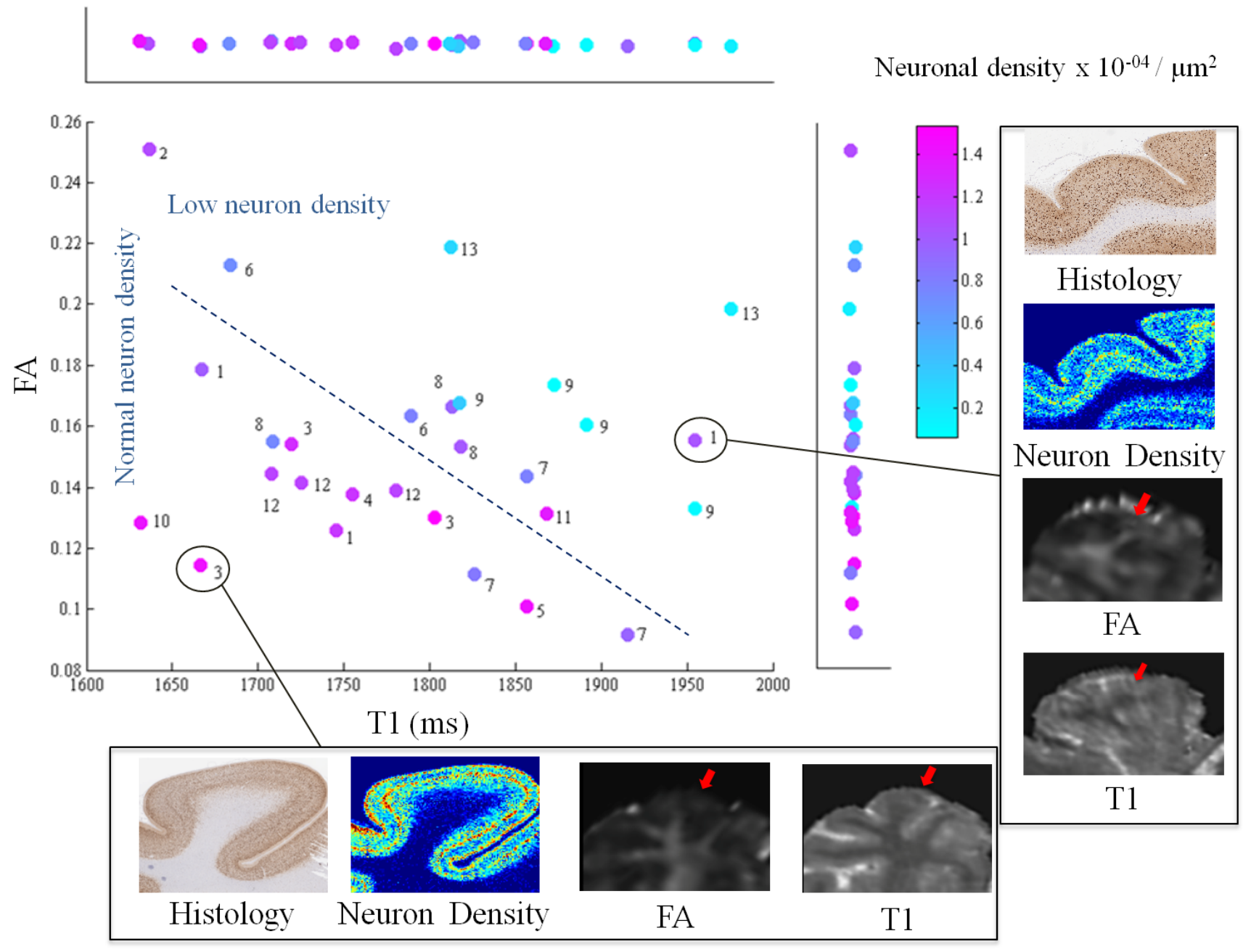



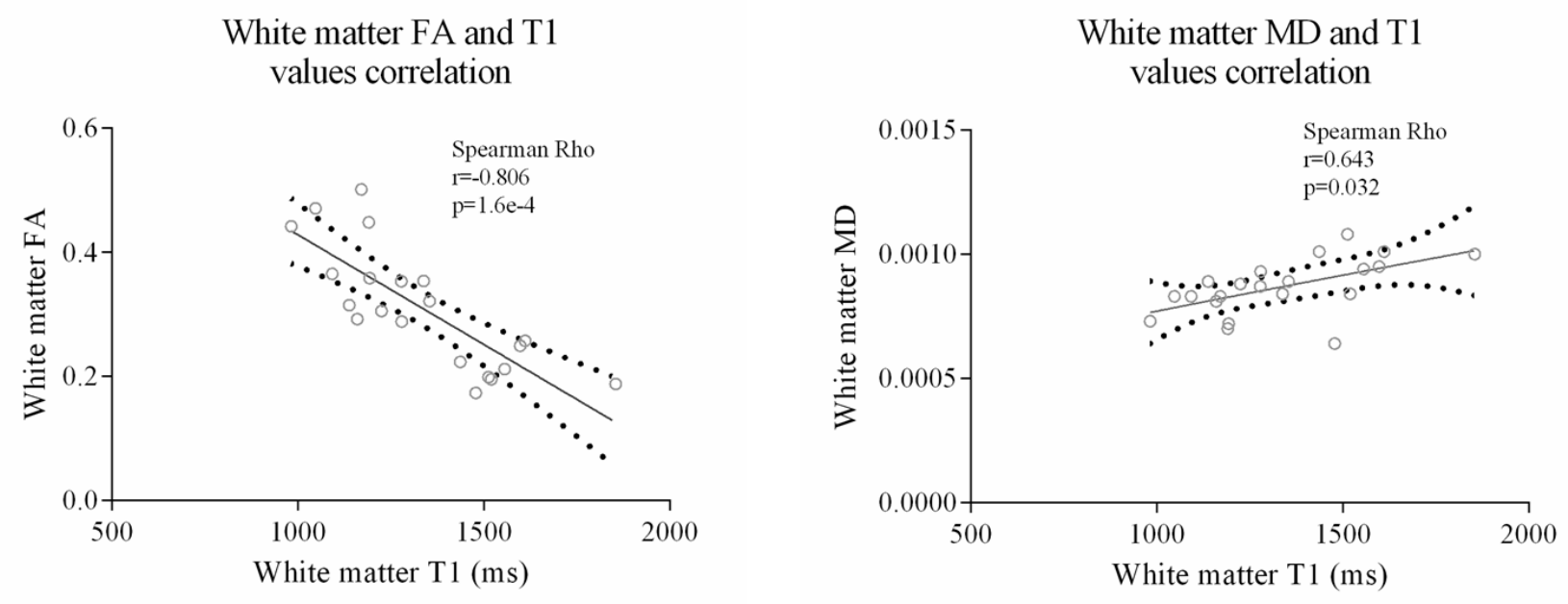

Grey matter neuronal density and NEUN field fraction correlation

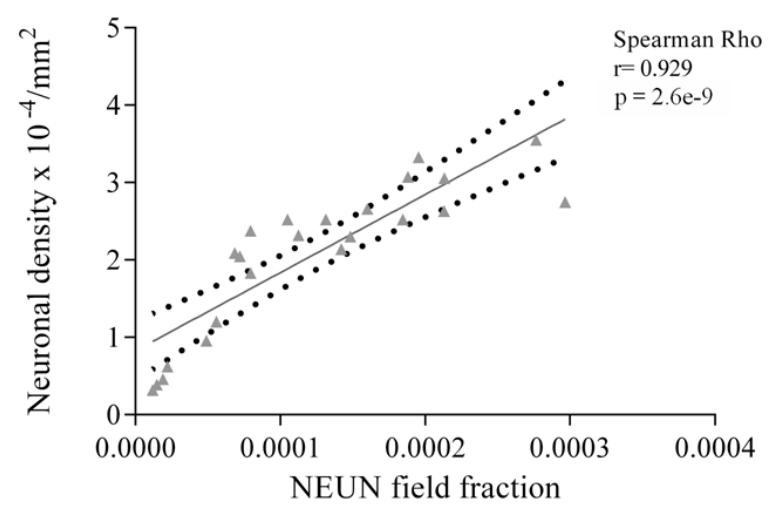




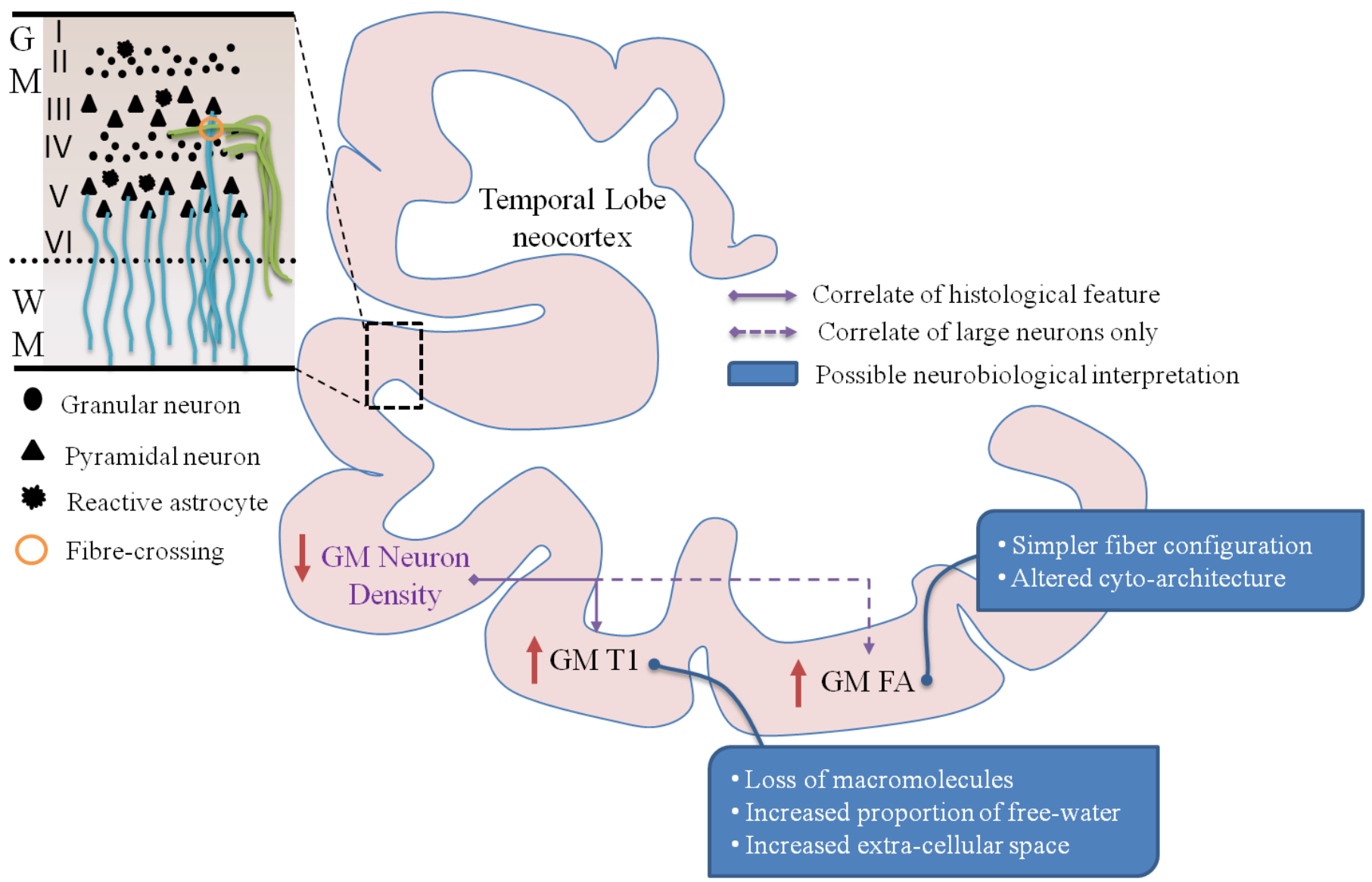

